\title{
Proton capture elements in the globular cluster NGC 2808
}

\section{First detection of large variations in sodium abundances along the Red Giant Branch}

\author{
E. Carretta ${ }^{1}$, A. Bragaglia ${ }^{2}$, C. Cacciari $^{2}$, and E. Rossetti ${ }^{2}$ \\ 1 INAF - Osservatorio Astronomico di Padova, vicolo dell'Osservatorio 5, 35122 Padova, Italy \\ 2 INAF - Osservatorio Astronomico di Bologna, via Ranzani 1, 40127 Bologna, Italy
}

Received 10 June 2003 / Accepted 14 August 2003

\begin{abstract}
We have used spectra obtained as part of the Science Verification program of the FLAMES multi-object spectrograph mounted on Kueyen (VLT-UT2) to perform an abundance analysis of stars along the giant branch (RGB) in the globular cluster NGC 2808. Sodium abundances are derived from Na D lines for a sample of 81 cluster stars spanning a range of about 2 mag from the tip of the RGB. Our results show that a large star-to-star scatter does exist at all positions along the RGB, suggesting large variations in the abundance of proton capture elements down to luminosities comparable to the red horizontal branch (HB). The distribution of Na abundances along the RGB seems to point out that in this cluster most of the observed spread has a primordial origin. Overimposed evolutionary effects, if any, must be only at "noise" level, at odds with results from a similar analysis in M 13 (Pilachowski et al. 1996). This study is a first step towards ascertaining if a link exists between the distribution of chemical anomalies in light elements along the RGB and the global properties of globular clusters, in particular the HB morphology.
\end{abstract}

Key words. stars: abundances - stars: evolution - stars: Population II - galaxy: globular clusters: general galaxy: globular clusters: individual: NGC 2808

\section{Introduction}

For a long time the globular clusters (GC) have been considered the best approximation in the Universe of Simple Stellar Populations (SSP), i.e. associations of single, coeval stars sharing the same initial chemical composition. These assumptions have been questioned in the last few years. Apart from the relevant presence of binary stars in GC's (showing up as Blue Stragglers or eclipsing binaries or peculiar objects, and strongly needed by dynamical considerations as a crucial ingredient in the dynamical evolution of clusters), also the other two properties that define a SSP have been seriously challenged on the basis of modern, accurate studies of the chemical composition of cluster stars.

Leaving aside the very peculiar case of $\omega$ Centauri, it has been evident for more than 30 years (Osborn 1971) that the hypothesis of monometallicity holds for GC only as far as "heavy" elements (those belonging to the Fe group) are concerned. Early studies (see the comprehensive reviews by Smith 1987 and Kraft 1994) based on indexes from photometry or low

Send offprint requests to: E. Carretta,

e-mail: carretta@pd.astro.it

* Based on data collected at the European Southern Observatory, Chile, during the FLAMES Science Verification. dispersion spectroscopy clearly demonstrated that the lighter elements (in primis carbon and nitrogen) showed marked differences along the RGB in several GC's. Moreover, striking variations in the $\mathrm{CH}$ band strenghts, anticorrelated with $\mathrm{CN}$ (and $\mathrm{NH}$, when accessible) strenghts were observed in several nearby clusters as far down as to main sequence and turn-off stars (see e.g. Cannon et al. 1998 and references therein)

Three fundamental steps forward have been made, thanks to the continuous progress in the building of efficient spectrographs: (i) since the early '90s, the high resolution studies by the Lick/Texas group (see Ivans et al. 2001 for updated references) well assessed that $\mathrm{Na}$ and $\mathrm{O}$ abundances are anticorrelated among the first ascent red giant branch (RGB) stars in almost all the clusters surveyed, for at least 1 magnitude below the RGB tip; (ii) the anomalies seem to be restricted to cluster stars, while the abundance pattern of field stars is perfectly explained by the classical first dredge-up and a second mixing episode after the level of the RGB-bump (Gratton et al. 2000); (iii) the $\mathrm{Na}-\mathrm{O}$ anticorrelation was recently found for the first time among turn-off stars in NGC 6752 by Gratton et al. (2001). These seminal works point out that not only the $\mathrm{CN}$-cycle (easily detectable even with low resolution analysis), but also the ON-cycle of the complete $\mathrm{CNO} \mathrm{H}$-burning cycle is at work, producing the observed pattern. In particular, $\mathrm{Na}$ can 
be produced from ${ }^{22} \mathrm{Ne}$ by the proton-capture fusion mechanism that is at work in the high temperature (inner) regions where $\mathrm{O}$ is transformed into N (see Denisenkov \& Denisenkova 1990; Langer et al. 1993).

The Na-O anticorrelation is the most convincing evidence of this process that, however, is restricted only to high density environments (such as those in the globular clusters), since Gratton et al. (2000) showed that $\mathrm{Na}$ and $\mathrm{O}$ abundances are hardly involved in the normal evolution along the RGB of isolated field stars. In a still unknown way, the large number of interactions between stars in GCs has to be involved in producing the observed pattern of chemical abundances.

Finally, the recent analysis of high resolution high quality UVES spectra of turn-off and early subgiant stars in NGC 6397 and NGC 6752 (Gratton et al. 2001) and 47 Tuc (Carretta et al. 2003a) indicated that groups of both Na-rich/O-poor and Napoor/O-rich unevolved stars are present in all these clusters. In turn, this must be due to pre-existing abundance variations, since these stars do not have the requirements (central high temperatures and an extended envelope able to bring the products of proton fusion to the photosphere) to produce the observed chemical pattern as a consequence of internal mixing during their evolution.

Primordial variations, viable to explain the observed anomalies, have been suggested both in the primordial gas of the proto-cluster (see Cottrell \& Da Costa 1981) in the very early phases of star/cluster formation, and as Na-rich, O-poor material ejected from a first generation of (now extinct) intermediate mass AGB stars that polluted the gas from which a second generation of stars (that are presently observed) formed (D'Antona et al. 1983; Ventura et al. 2001). Notice that in this latter case the assumption of strictly coeval stars in a GC must be dropped.

On the other hand, the increasingly high $\mathrm{N}$ abundances, accompanied by a progressive decline in $\mathrm{C}$ abundance and ${ }^{12} \mathrm{C} /{ }^{13} \mathrm{C}$ ratio, observed at increasing luminosity along the RGB in many clusters, testifies that changes are going on during the evolution of stars, such as e.g. very deep mixing in RGB stars, that may be triggered by enhanced core rotation possibly due to the dense cluster environment.

Nowadays, the most likely explanation of the overall chemical pattern observed in GC stars is that a contribution of both aspects (primordial and evolutionary mixing) is required. The debate seems presently shifted rather on how to disentangle these two contributions and to properly ascertain their relative weights within a cluster and in clusters of different physical properties (Horizontal Branch (HB) morphology, density, age, metallicity, etc.).

To this purpose large and homogeneous sets of cluster stars must be observed, possibly in different evolutionary stages. This has become possible only recently, with the advent of efficient multiobject spectrographs on large telescopes, such as Hydra at the KPNO 4-m and WIYN $3.5 \mathrm{~m}$ telescopes used by Pilachowski et al. (1996b) and Sneden et al. (2000) to survey the RGB in M 13, or in M 15 and M 92 respectively.

FLAMES mounted at the $8 \mathrm{~m}$ Kueyen VLT-UT2 telescope is the ideal instrument for this type of studies (see Pasquini et al. 2002 for a technical description).
NGC 2808 is by itself a very interesting object, since its HB morphology (a red stubby HB and a population of blue HB stars, about $1 / 4$ of the total HB stars) has often been labelled as the second parameter effect working within an individual cluster. However, the overwhelming majority of previous works was photometric, to investigate the morphology of the color-magnitude diagram (CMD). The only spectroscopic study is that of Gratton (1982), who analyzed a single giant star obtaining a metallicity of $[\mathrm{Fe} / \mathrm{H}]=-1.1 \mathrm{dex}^{1}$.

This situation dramatically changed at the end of January 2003, when $\sim 130$ RGB stars in NGC 2808 were observed during the program "Mass loss in Globular Cluster Red Giant Stars" (proposed by C. Cacciari and A. Bragaglia), that was part of the FLAMES Science Verification program. The results of this study are presented in a separate paper (Cacciari et al. 2003). In the present paper we exploit part of this material for the analysis of the sodium D lines in order to get insights on the distribution of this proton capture element along the RGB.

In the next section we present the star selection (that was performed for studying the mass loss process and was not optimized for the present purpose) and the observations. In Sects. 3 and 4 , respectively, we explain the atmospheric parameters adopted and the abundance analysis for $\mathrm{Na}$, whose results are discussed in Sect. 5. Comparison with results for other clusters are made in Sect. 6, and a short summary is given in Sect. 7.

\section{Sample selection and observations}

Our program stars were observed in 2003, January 24-25 during the FLAMES Science Verification program at the ESO Paranal Observatory. The data were released to the public on 2003, March 3. The night was not photometric, the sky conditions were thin cirrus and the seeing was about 0.9 arcsec. 86 stars were observed with the grating HR 12 of GIRAFFE, giving an useful spectral range from 5820 to $6134 \AA$, with a resolution of about 15000 at the centre of spectra. During all GIRAFFE exposures, the available fibers ( 8 for each setup, including one fiber on the sky) feeding the Red Arm of the high resolution spectrograph UVES were centered on other RGB stars, for a total of 20 objects observed at high resolution ( $R \sim 45000)$. Only 4 stars were observed by both GIRAFFE and UVES. Details of these UVES observations and their analysis are deferred to a forthcoming paper (Carretta et al. 2003b). Here, we concentrate only on the GIRAFFE sample in the $\mathrm{Na}$ $D$ region.

Figure 1 indicates the position of our program stars in the $V, B-V$ colour-magnitude diagram of NGC 2808. We remind here that the main purpose of this program was to study the mass loss along the RGB, so the observations are concentrated in the region close to the RGB tip. Cool and (moderately) metal-rich stars are not the best targets for abundance analysis due to the line crowding, affecting the continuum positioning,

\footnotetext{
${ }^{1}$ We use the usual spectroscopic notation: $\log n(\mathrm{~A})$ is the abundance (by number) of the element $\mathrm{A}$ in the usual scale where $\log n(\mathrm{H})=12$; notation $[\mathrm{A} / \mathrm{H}]$ is the logarithmic ratio of the abundances of elements $\mathrm{A}$ and $\mathrm{H}$ in the star, minus the same quantity in the Sun.
} 

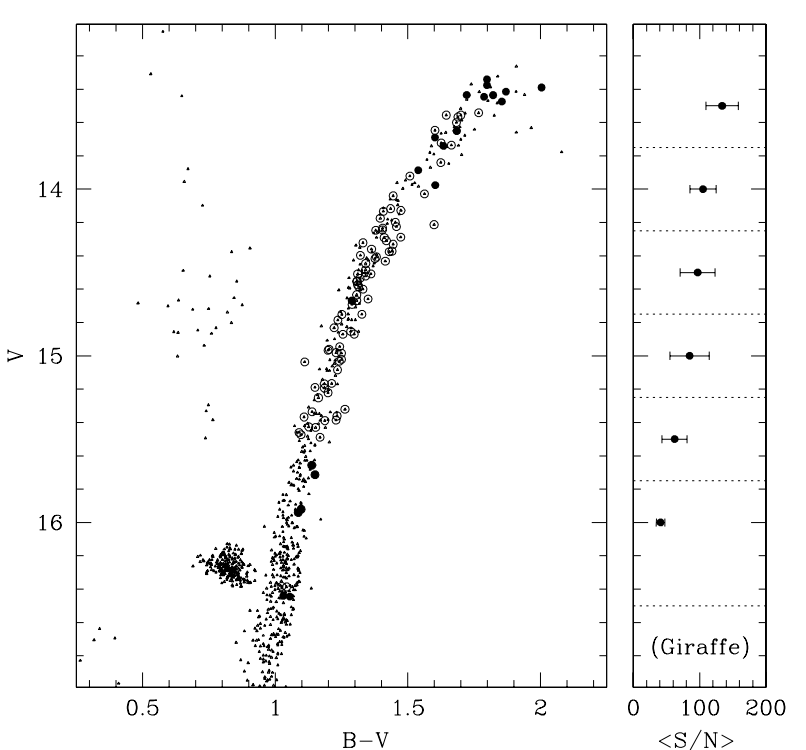

Fig. 1. Left panel: $V, B-V$ colour-magnitude diagram of NGC 2808 (Piotto et al. 2003, in preparation). Stars observed with the GIRAFFE HR 12 grating in the Medusa configuration are indicated by open circles, while filled circles are stars observed with fibers feeding the UVES Red Arm. Right panel: average $S / N$ in 0.5 mag bins along the RGB.

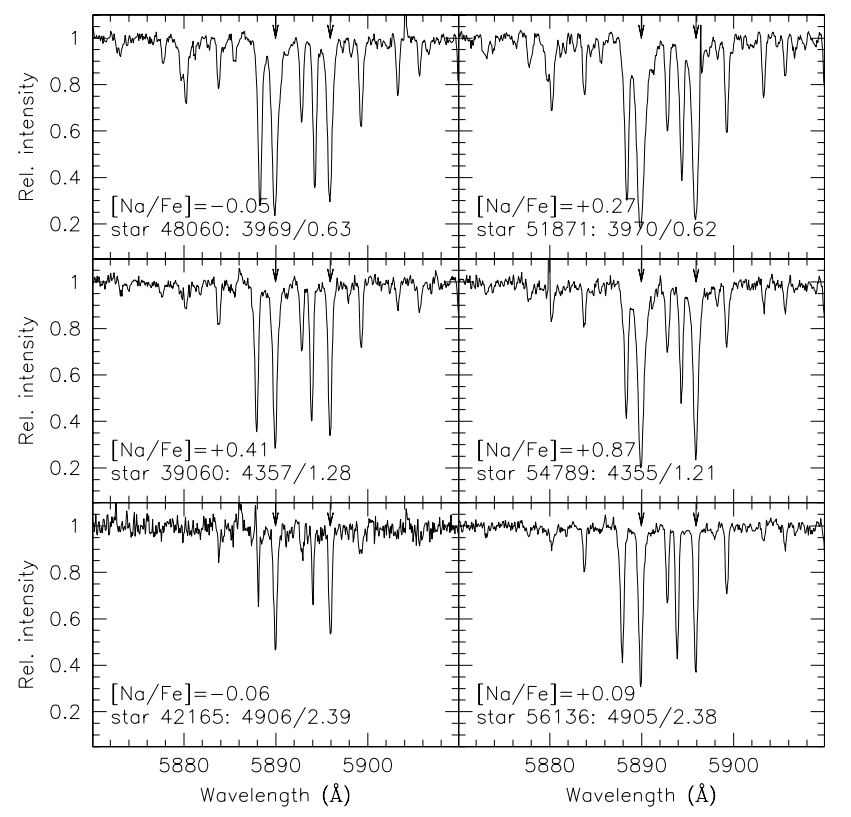

Fig. 2. Typical spectra for bright (upper panels), intermediate (middle panels) and faint (lower panels) objects in our sample with different $\mathrm{Na}$ abundances. The small arrows indicate the position of the stellar Na I D lines; notice how well they can be separated from the interstellar lines, here shifted to the blue. We also indicate for each star the values of $T_{\text {eff }}, \log g$, and $[\mathrm{Na} / \mathrm{Fe}] . S / N$ (per pixel) varies from about 100 , to 85 , to 45 for the three magnitude levels.

and to concerns on existing model atmospheres that are unable to well reproduce the cooler red giants.

In Fig. 2 typical spectra are displayed for stars spanning all the range of parameters sampled along the RGB.
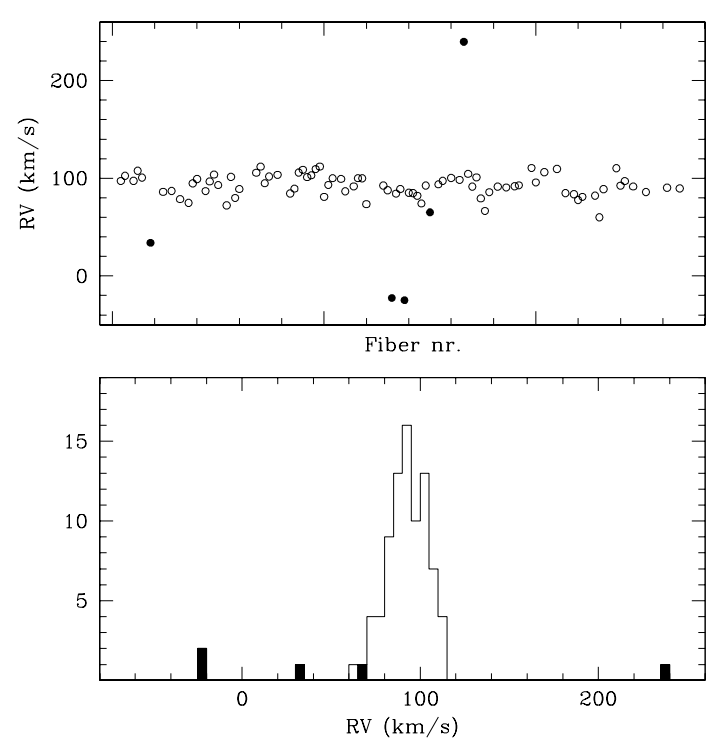

Fig. 3. Upper panel: measured radial velocities for program stars plotted as a function of the fiber numbers. Filled circles are stars rejected from our analysis as non-members. Lower panel: histogram of the radial velocities, where the rejected stars are indicated by the filled histogram.

The sample goes from the tip of the RGB (about $M_{V}=-3$, adopting a distance modulus $(m-M)_{V}=15.59$ from Harris 1996) to just below the level of the red HB (about $M_{V}=1$ ). Only two stars have been observed below $V=16$, the bulk of program stars lie within 2 mag from the RGB tip. The right panel in Fig. 1 shows how the average $S / N$ ratio varies as a function of the $V$ magnitude along the RGB. The scatter around a mean value at fixed magnitude is likely due to a combination of small mis-positioning errors of the star in the individual fibers and different throughtputs in transmission from different fibers.

Cluster membership was mostly secured by choosing stars near the mean ridge line of the giant branch and checked a posteriori by measuring radial velocities from spectral lines. The radial velocity $(R V)$ for each star was derived by fitting Gaussian profiles to about 30 lines (with the IRAF ${ }^{2}$ routine rvidlines).

Figure 3 shows the distribution of radial velocities. A small zero point offset between fibers is possible, due to the reduction procedure not optimized for precise radial velocities measurements, that could reach $\pm 10 \mathrm{~km} \mathrm{~s}^{-1}$, according to the ESO relevant web pages. We have not done any detailed check on radial velocities, since it was outside our main purpose; we only note that the 4 stars that were observed also with UVES at high resolution - and more reliable wavelength calibration - show a difference between derived velocities from 1 to $-8 \mathrm{~km} \mathrm{~s}^{-1}$, with an average of $-3.5 \mathrm{~km} \mathrm{~s}^{-1}$, and measurement of the telluric Na I line at $5889.884 \AA$ in star \#56136 indicates a velocity zero point of $3.5 \mathrm{~km} \mathrm{~s}^{-1}$. So the derived $R V$ 's are in general precise enough to discriminate between cluster and field stars. Using the $R V$ distribution, we disregarded 4 stars as sure field

${ }^{2}$ IRAF is distributed by the NOAO, which are operated by AURA, under contract with NSF. 
interlopers. Another star was rejected from the sample after the abundance analysis (see below, Sect. 4). The average heliocentric velocity is $R V=102.1 \mathrm{~km} \mathrm{~s}^{-1}(\sigma=10.9)$, after eliminating these 5 non-members.

The final sample consists therefore in 81 red giant stars. What is the physical evolutionary status of these giants? Are they truly first ascent giant stars or is the sample contaminated by stars in a more evolved stage, i.e. Asymptotic Giant Branch (AGB) stars? For the brightest objects there is no way to discriminate between RGB and AGB stars either photometrically or spectroscopically: their position in the CMD is almost identical, and small differences in colours may well be due to photometric errors and/or variability, and in both cases emissions on the $\mathrm{H} \alpha$ wings can be present. Anyway, from robust evolutionary theory expectations, AGB stars should represent only a small fraction $(\sim 10 \%)$ of the brightest objects. When we consider lower luminosity stars, photometric distinction is easier, and we may also hope to detect AGB stars via $\mathrm{H} \alpha$ emission wings (as done by Sneden et al. 2000): this emission, which is rather common also in RGB stars near the tip (see e.g. Lyons et al. 1996, and the vast literature cited there), is not present in fainter objects. Hence, if stars with $M_{V} \lesssim-1$ or fainter present $\mathrm{H} \alpha$ emission wings, they may be taken as - or at least suspected to be - AGB stars. Cacciari et al. (2003) have done a similar survey of the same stars we are analysing here, and detected emissions only for one such star (\#10341): we will still retain it in our sample, cautioning that it could well not be a true RGB star.

\section{Atmospheric parameters}

As described in Cacciari et al. (2003), the effective temperatures $T_{\text {eff }}$ and bolometric corrections B.C. for our stars were derived from the $V-K$ colors in the 2MASS All-Sky Data Release (accessible at www.ipac.caltech.edu/2mass/releases/ allsky/ and released on March 25, 2003), and using the calibrating equations by Alonso et al. (1999, with the erratum of Alonso et al. 2001). We have adopted $A_{K}=0.353 E(B-V)$ and $A_{V}=3.1 E(B-V)$ from Cardelli et al. (1989), and $E(B-V)=0.22$ from Harris (1996).

The 2MASS photometry was transformed to the TCS system and an input metallicity of $[\mathrm{Fe} / \mathrm{H}]=-1.25$ was adopted $^{3}$.

Surface gravities $\log g$ were obtained from effective temperatures and bolometric corrections, using the distance modulus $(m-M)_{V}=15.59$, and assuming that the stars have masses of $0.85 M_{\odot}$. The adopted bolometric magnitude of the Sun is $M(\mathrm{bol})_{\odot}=4.75$.

For the overall metallicity, we adopted the average metal abundance as derived by the analysis of the high resolution UVES red spectra (Carretta et al. 2003b): $[\mathrm{A} / \mathrm{H}]=-1.14 \pm$ $0.01 \mathrm{dex}, \sigma=0.06 \operatorname{dex}(19 \mathrm{stars})^{4}$. This choice stems from the

\footnotetext{
3 This value is intermediate among several previous determinations (cf. Walker 1999), and is slightly different from the metallicity we adopt in the present analysis; however, the dependence of $(V-K)$ on $[\mathrm{Fe} / \mathrm{H}]$ is so weak that temperatures are almost unaffected by this difference.

${ }^{4}$ This in turn is based on a solar iron abundance of $\log n(\mathrm{Fe} \mathrm{I})_{\odot}=7.54$, see Gratton et al. (2001).
}

small number of Fe I lines available in our spectral range (about 17 lines, on average, are measurable per star) and from the rather low resolution. As discussed, e.g., in Carretta \& Gratton (1997), at lower resolution line blends are more frequent, leading to overestimate the equivalent widths (EW) in a complex way that is a function of metallicity, $S / N$ and temperature (all affecting the line crowding), as well as resolution. Since the RGB magnitude range monitored by GIRAFFE observations was covered also by UVES observations, we believe that the use of UVES-based metallicities is justified and does not bias the results we obtain from GIRAFFE data.

Finally, based on the same considerations, we adopted the microturbulent velocity $v_{t}$ derived from the high resolution UVES spectra, using a large number of Fe I lines of different strenghts. The resulting relation is well constrained, so we adopted $v_{t}=1.73 \mathrm{~km} \mathrm{~s}^{-1}$ for $T_{\text {eff }}<4100 \mathrm{~K}$ and $v_{t}=$ $-1.3 \times 10^{-4} \times T_{\text {eff }}+7.10$ for $T_{\text {eff }} \geq 4100$.

Star designations and derived atmospheric parameters are listed in the first four columns of Table 1.

\section{Analysis: Sodium abundances}

Equivalent widths (EW) of Na D lines were measured following the method outlined in Bragaglia et al. (2001): we refer to that paper for further details. The fraction of the (highest) spectrum points to be used by the automatic program in order to set a local continuum centered on the feature of interest is the crucial point of the procedure. We checked this parameter by inspecting by eye the continuum location as defined by the program for some lines of typical spectra distributed all over the temperature range. After this test, we set this fraction to $1 / 3$ for stars with $T_{\text {eff }} \leq 4200 \mathrm{~K}$ and to $1 / 2$ for warmer stars.

Using this value and the atmospheric parameters derived in the previous section, we measured the EWs of the Na D lines at 5889.97 and $5894.94 \AA$ for all 81 program stars, and derived the $\mathrm{Na}$ abundances interpolating in the Kurucz (1995) grid of model atmospheres with the overshooting option set on.

However, a Gaussian function is a poor approximation for fitting these very strong lines and may give misleading results, missing the relevant contribution of the damping wings of the lines. For these reasons we decided to adopt the $\mathrm{Na}$ abundances obtained from comparison with synthetic D1 and D2 lines: the procedure is much more time consuming but gives more reliable results than a Gaussian fit or even a direct integration. Moreover, concerns related to the positioning of the continuum are much reduced.

Hence, we computed a grid of synthetic spectra for each of the Na D lines. Each grid includes 21 spectra with $T_{\text {eff }}$ ranging from 3950 to $4950 \mathrm{~K}$ (this range brackets all our program stars) in steps of $50 \mathrm{~K}$. Using the single value $[\mathrm{A} / \mathrm{H}]=-1.14 \mathrm{dex}$, $v_{t}$ for each synthetic spectrum was computed using the relation obtained from the UVES spectra, as described in the previous section; the gravity was derived from a $T_{\text {eff }}-\log g$ relation obtained with a linear fit from our program stars.

For each of the 21 spectra (i.e. values of $T_{\text {eff }}$ ) in our grid we computed 10 synthetic spectra of the $\mathrm{Na}$ line by varying the $[\mathrm{Na} / \mathrm{Fe}]$ ratio from -0.4 to $+1.4 \mathrm{dex}$, in steps of 0.2 dex. Thus we could associate to each $\mathrm{Na}$ line a set of 210 synthetic spectra 
Table 1. Atmospheric parameters and derived $\mathrm{Na}$ abundances, both in LTE and in NLTE. Star designations are taken from Piotto et al. (2003, in preparation).

\begin{tabular}{|c|c|c|c|c|c|c|}
\hline Star & $T_{\mathrm{eff}}$ & $\log g$ & $\mathrm{~km} \mathrm{~s}^{-1}$ & $\begin{array}{c}{[\mathrm{Na} / \mathrm{Fe}]} \\
\mathrm{LTE} \\
\operatorname{dex}\end{array}$ & $\begin{array}{c}{[\mathrm{Na} / \mathrm{Fe}]} \\
\mathrm{NLTE} \\
\operatorname{dex} \\
\end{array}$ & dex \\
\hline 7536 & 4245 & 1.18 & 1.58 & -0.16 & +0.06 & 0.04 \\
\hline 7558 & 4691 & 1.87 & 1.00 & +0.17 & +0.28 & 0.09 \\
\hline 7788 & 4459 & 1.53 & 1.30 & +0.12 & +0.29 & 0.15 \\
\hline 8603 & 4290 & 1.24 & 1.52 & -0.24 & -0.02 & 0.13 \\
\hline 8739 & 4211 & 1.12 & 1.63 & -0.08 & +0.14 & 0.15 \\
\hline 8826 & 4564 & 1.66 & 1.17 & +0.42 & +0.57 & 0.16 \\
\hline 9230 & 4132 & 0.96 & 1.73 & +0.04 & +0.29 & 0.02 \\
\hline 10012 & 4243 & 1.12 & 1.58 & +0.23 & +0.45 & 0.04 \\
\hline 10105 & 4455 & 1.44 & 1.31 & -0.05 & +0.13 & 0.01 \\
\hline 10201 & 4717 & 2.02 & 0.97 & +0.42 & +0.52 & 0.08 \\
\hline 10265 & 4283 & 1.18 & 1.53 & +0.24 & +0.45 & 0.04 \\
\hline 10341 & 4399 & 1.40 & 1.38 & +0.44 & +0.63 & 0.04 \\
\hline 10571 & 4252 & 1.19 & 1.57 & -0.01 & +0.21 & 0.08 \\
\hline 13575 & 4569 & 1.80 & 1.16 & -0.23 & -0.10 & 0.01 \\
\hline 13983 & 4826 & 2.17 & 0.83 & +0.35 & +0.42 & 0.16 \\
\hline 30523 & 4733 & 1.94 & 0.95 & +0.26 & +0.37 & 0.05 \\
\hline 30900 & 4666 & 1.74 & 1.03 & +0.21 & +0.34 & 0.11 \\
\hline 31851 & 4731 & 1.83 & 0.95 & +0.77 & +0.89 & 0.03 \\
\hline 32469 & 4379 & 1.14 & 1.41 & +0.44 & +0.65 & 0.11 \\
\hline 32685 & 4788 & 2.03 & 0.88 & +0.37 & +0.47 & 0.01 \\
\hline 32862 & 4525 & 1.69 & 1.22 & +0.15 & +0.30 & 0.10 \\
\hline 32909 & 4572 & 1.66 & 1.16 & +0.29 & & 0.09 \\
\hline 32924 & 4538 & 1.57 & 1.20 & +0.54 & +0.70 & 0.15 \\
\hline 33918 & 4312 & 1.21 & 1.49 & +0.06 & +0.27 & 0.09 \\
\hline 35265 & 4547 & 1.76 & 1.19 & +0.07 & +0.21 & 0.10 \\
\hline 37496 & 4547 & 1.58 & 1.19 & +0.26 & +0.42 & 0.03 \\
\hline 37998 & 4317 & 1.18 & 1.49 & +0.12 & +0.34 & 0.08 \\
\hline 38228 & 4588 & 1.65 & 1.14 & +0.38 & +0.52 & 0.01 \\
\hline 38244 & 4659 & 1.78 & 1.04 & +0.23 & +0.36 & 0.04 \\
\hline 38967 & 4717 & 1.83 & 0.97 & +0.41 & +0.53 & 0.08 \\
\hline 39060 & 4357 & 1.28 & 1.44 & +0.21 & & 0.08 \\
\hline 40983 & 4364 & 1.26 & 1.43 & +0.29 & +0.49 & 0.01 \\
\hline 41008 & 4684 & 1.79 & 1.01 & +0.67 & +0.80 & 0.03 \\
\hline 41828 & 4409 & 1.36 & 1.37 & +0.11 & +0.30 & 0.22 \\
\hline 41969 & 4377 & 1.30 & 1.41 & +0.07 & +0.26 & 0.01 \\
\hline 42073 & 4268 & 1.17 & 1.55 & -0.02 & +0.20 & 0.13 \\
\hline 42165 & 4906 & 2.39 & 0.72 & -0.09 & -0.06 & 0.04 \\
\hline 42789 & 4813 & 1.93 & 0.84 & +0.78 & +0.89 & 0.09 \\
\hline 42886 & 4791 & 2.14 & 0.87 & +0.11 & +0.19 & 0.08 \\
\hline 42996 & 4769 & 1.93 & 0.90 & +0.29 & +0.39 & 0.09 \\
\hline 43041 & 4035 & 0.70 & 1.73 & -0.23 & +0.05 & 0.04 \\
\hline 43247 & 4463 & 1.50 & 1.30 & +0.29 & +0.46 & 0.09 \\
\hline 43794 & 4431 & 1.38 & 1.34 & +0.05 & +0.24 & 0.13 \\
\hline 43822 & 4411 & 1.39 & 1.37 & +0.16 & +0.34 & 0.03 \\
\hline 44665 & 4310 & 1.18 & 1.50 & +0.17 & +0.39 & 0.16 \\
\hline 44984 & 4405 & 1.36 & 1.37 & -0.00 & +0.19 & 0.13 \\
\hline 45443 & 4425 & 1.39 & 1.35 & +0.05 & +0.23 & 0.13 \\
\hline
\end{tabular}

Table 1. continued.

\begin{tabular}{lcccccc}
\hline \hline Star & $T_{\text {eff }}$ & $\log g$ & $v_{t}$ & {$[\mathrm{Na} / \mathrm{Fe}]$} & {$[\mathrm{Na} / \mathrm{Fe}]$} & $\sigma$ \\
& & & & LTE & NLTE & \\
& $(\mathrm{K})$ & dex & $\mathrm{km} \mathrm{s}^{-1}$ & dex & dex & dex \\
\hline 45840 & 4220 & 1.06 & 1.61 & -0.14 & +0.10 & 0.03 \\
46041 & 4568 & 1.63 & 1.16 & +0.26 & +0.41 & 0.04 \\
46367 & 4383 & 1.46 & 1.40 & +0.21 & +0.38 & 0.03 \\
46663 & 4474 & 1.41 & 1.28 & +0.47 & +0.65 & 0.04 \\
46868 & 4463 & 1.50 & 1.30 & +0.05 & +0.22 & 0.02 \\
47145 & 4051 & 0.74 & 1.73 & +0.02 & +0.29 & 0.05 \\
47421 & 4288 & 1.14 & 1.53 & +0.21 & +0.43 & 0.11 \\
48011 & 4375 & 1.28 & 1.41 & +0.63 & +0.83 & 0.01 \\
48060 & 3969 & 0.63 & 1.73 & -0.35 & -0.05 & 0.04 \\
48128 & 4368 & 1.31 & 1.42 & +0.48 & +0.68 & 0.08 \\
49509 & 4164 & 0.94 & 1.69 & +0.12 & +0.37 & 0.08 \\
49680 & 3952 & 0.62 & 1.73 & -0.37 & -0.07 & 0.05 \\
49743 & 4665 & 1.77 & 1.04 & +0.46 & +0.60 & 0.06 \\
49942 & 4023 & 0.75 & 1.73 & -0.15 & +0.13 & 0.02 \\
50371 & 4054 & 0.82 & 1.73 & -0.23 & +0.04 & 0.07 \\
50861 & 4039 & 0.69 & 1.73 & +0.13 & +0.41 & 0.06 \\
50866 & 4432 & 1.42 & 1.34 & +0.40 & +0.58 & 0.09 \\
50910 & 4577 & 1.64 & 1.15 & +0.25 & +0.40 & 0.02 \\
51416 & 4546 & 1.61 & 1.19 & +0.39 & +0.54 & 0.09 \\
51515 & 4154 & 1.05 & 1.70 & +0.04 & +0.28 & 0.06 \\
51646 & 4886 & 2.01 & 0.75 & +0.88 & +0.98 & 0.08 \\
51871 & 3970 & 0.62 & 1.73 & -0.02 & +0.27 & 0.10 \\
51963 & 4242 & 1.11 & 1.59 & +0.06 & +0.28 & 0.04 \\
52006 & 4397 & 1.27 & 1.38 & +0.62 & +0.81 & 0.09 \\
54264 & 4668 & 1.84 & 1.03 & +0.15 & +0.27 & 0.09 \\
54284 & 4327 & 1.26 & 1.47 & +0.14 & +0.34 & 0.11 \\
54733 & 4236 & 1.08 & 1.59 & +0.25 & +0.48 & 0.05 \\
54789 & 4355 & 1.21 & 1.44 & +0.66 & +0.87 & 0.01 \\
55031 & 3995 & 0.67 & 1.73 & -0.35 & -0.05 & 0.05 \\
55437 & 4440 & 1.44 & 1.33 & +0.27 & +0.45 & 0.05 \\
55609 & 4430 & 1.46 & 1.34 & +0.23 & +0.41 & 0.04 \\
55627 & 4459 & 1.41 & 1.30 & +0.52 & +0.71 & 0.13 \\
56136 & 4905 & 2.38 & 0.72 & +0.04 & +0.09 & 0.09 \\
\hline 5710 & 4643 & 1.86 & 1.06 & +0.04 & +0.16 & 0.09 \\
\hline & & & & & &
\end{tabular}

spanning the relevant range both in temperature and $\mathrm{Na}$ abundance. These synthetic spectra were convolved with a Gaussian function to match the resolution of the GIRAFFE spectra. We found that a smoothing $F W H M$ of $0.3 \AA$ is appropriate for our program stars.

Starting with input $\mathrm{Na}$ abundances determined by line analysis, we chose the nearest value of temperature for each star, and compared the observed spectrum to the 3 synthetic spectra bracketing the $\mathrm{Na}$ abundance from EW. Visual inspection was used to obtain the $\mathrm{Na}$ abundances from each line. For the star $\mathrm{Na}$ abundance we then took simply the average from the two 


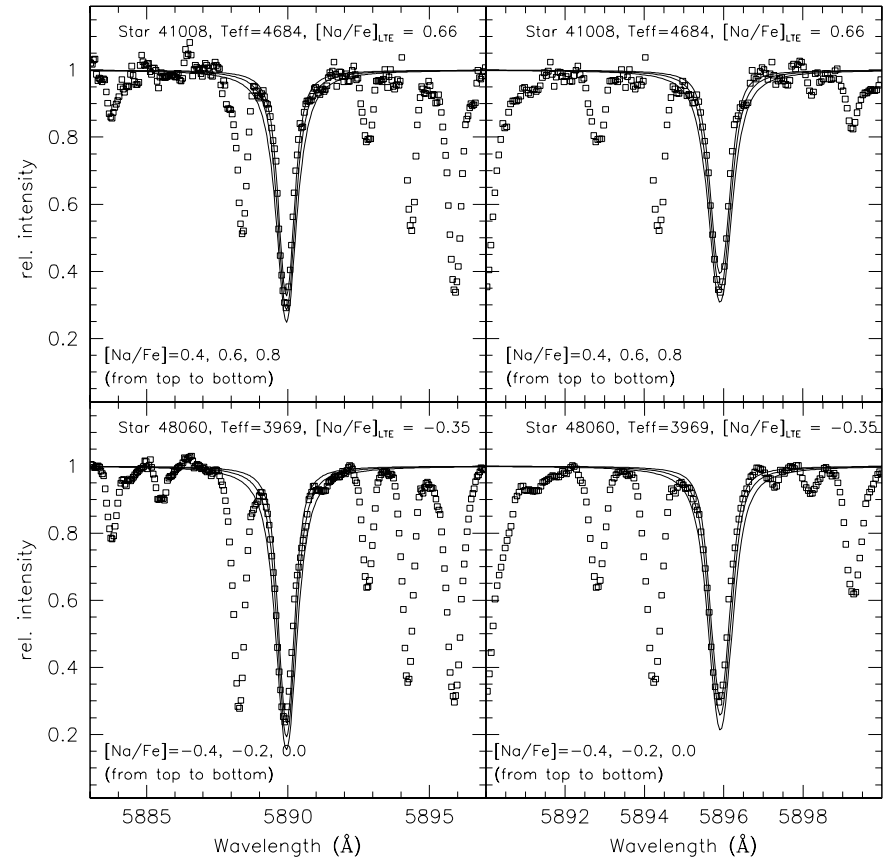

Fig. 4. Upper left panel: spectrum synthesis of the Na D2 line at $5889.97 \AA$ in star 41008. Open squares are the observed spectrum, while lines represent the synthetic spectra computed at $4700 \mathrm{~K}$ for 3 different $\mathrm{Na}$ abundances: $[\mathrm{Na} / \mathrm{Fe}]=0.4,0.6$ and 0.8 dex from top to bottom, respectively. Upper right panel: the same, but for the Na D1 line at $5895.94 \AA$. Lower left panel: spectrum synthesis of the Na D2 line in star 48060, near the RGB tip. The plotted synthetic spectra are computed at $3950 \mathrm{~K}$ and for abundance ratios of $[\mathrm{Na} / \mathrm{Fe}]=-0.4,-0.2$ and 0.0 from top to bottom. Lower right panel: the same, but for the Na D1 line. All synthetic spectra have been convolved with a Gaussian having $F W H M=0.3 \AA$ to take into account the instrumental profile of the observed spectra. All abundances are given in the LTE assumption.

$\mathrm{Na} \mathrm{D}$ lines, adopting $\log n(\mathrm{Na})=6.23$ as the solar $\mathrm{Na}$ abundance.

In Fig. 4 we show two examples of the fitting of synthetic spectra to the observed $\mathrm{Na} \mathrm{D}$ lines for two stars in different position along the RGB. In the upper panels, the star 41008 has $T_{\text {eff }}=4684 \mathrm{~K}$ and was compared to spectra computed at $4700 \mathrm{~K}$, while in the lower panels the program star has $T_{\text {eff }}=3969$ and $\mathrm{Na}$ abundances are obtained through comparison with synthetic spectra computed at $3950 \mathrm{~K}$.

This figure shows that despite the difference of $\sim 1 \mathrm{dex}$ in $\mathrm{Na}$ abundance and $\sim 800 \mathrm{~K}$ in temperature, the $\mathrm{Na}$ features are well reproduced.

A comparison of $\mathrm{Na}$ abundances derived by line analysis (EWs) and by spectrum synthesis is highly instructive. The average abundance difference (in the sense synthesis minus line) is $\Delta[\mathrm{Na} / \mathrm{Fe}]=+0.30 \pm 0.01$ dex, with an rms $=0.09$ dex $\left(81\right.$ stars $\left.^{5}\right)$. This test illustrates how severely the Gaussian

\footnotetext{
${ }^{5}$ We exclude from the sample and from the following discussion star 37781, that is probably not-member of the cluster even if its radial velocity is compatible with membership. The unusually high $\mathrm{Na}$ abundance obtained for this stars $([\mathrm{Na} / \mathrm{Fe}] \simeq+1.5)$ is likely due to an erroneous estimate of atmospheric parameters due to the initial assumption that this star is member of NGC 2808, or because it might be an AGB star.
}

fitting approximation may underestimate the measure of EWs for these strong lines, resulting in spurious $\mathrm{Na}$ abundances.

Since the observations were made for other purposes and did not aim at deriving chemical abundances, rapidly rotating early type stars were not observed. Lacking a suitable template to subtract telluric lines, we evaluated their impact on derived abundances by estimating their contribution to the EW of the $\mathrm{Na}$ lines within the region where the fitting of synthetic spectra was performed. We verified that the possible effect of telluric lines on the derived abundances amounts to no more than a few hundredths of a dex and was therefore considered negligible.

Finally, we neglected the hyperfine splitting for $\mathrm{Na} D$ lines. Explorative computations with atomic parameters for the components of each line taken from McWilliam et al. (1995) allowed us to conclude that the effect on our $\mathrm{Na}$ abundances is negligible, likely because in very strong lines the effect itself is reduced since all hyperfine components in the line core are saturated (see McWilliam et al. 1995).

\subsection{NLTE corrections}

Statistical equilibrium computations (e.g. Bruls et al. 1992; Gratton et al. 1999) show that the NLTE abundance corrections for $\mathrm{Na} \mathrm{D}$ lines follow a rather complicated pattern, due to the complex relative interplay between photoionization and the so-called photo suction effect (Bruls et al. 1992) that consists in a recombination cascade to the ground level, where the $\mathrm{D}$ lines originate. Moreover, the strong $\mathrm{Na} \mathrm{D}$ lines form high in the atmosphere, where departures from LTE are larger. In order to assess the true Na distribution, a careful evaluation of these effects is then required.

We derived appropriate corrections for departure from the LTE following the prescriptions of Gratton et al. (1999). Since these corrections are a function of both stellar physical status (temperature and gravity) and line strength, we measured the equivalent widths of synthetic Na D2 and D1 lines for all ( $T_{\text {eff }}$, $[\mathrm{Na} / \mathrm{Fe}])$ pairs. These EWs were used to construct interpolating relations (one for each line) as a function of temperature and $\mathrm{Na}$ abundance.

Next, we entered in these relations with stellar temperatures and previously derived LTE Na abundances from spectrum synthesis for all our program stars, deriving $[\mathrm{Na} / \mathrm{Fe}]$ ratios properly corrected for departure from LTE following Gratton et al. (1999). These corrected abundances are listed in Table 1 (labeled as $[\mathrm{Na} / \mathrm{Fe}]_{\mathrm{NLTE}}$ ) and represent our final set of abundances on which the following considerations and discussions are based. As reference for the reader, in this table are also listed abundances derived under the LTE assumption. The errors listed in Table 1 are the rms of the straight average between the abundances as given by $\mathrm{Na} \mathrm{D} 2$ and $\mathrm{D} 1$ lines.

In Fig. 5 we show the NLTE corrections to $[\mathrm{Na} / \mathrm{Fe}] \mathrm{LTE}$ abundances as a function of effective temperature. As one can see, the corrections are progressively larger going from lower luminosity giants toward giants near the tip of the RGB (where they can reach as much as $0.3 \mathrm{dex}$ ). Neglecting these corrections would then result in a spurious trend as a function of $T_{\text {eff }}$, 


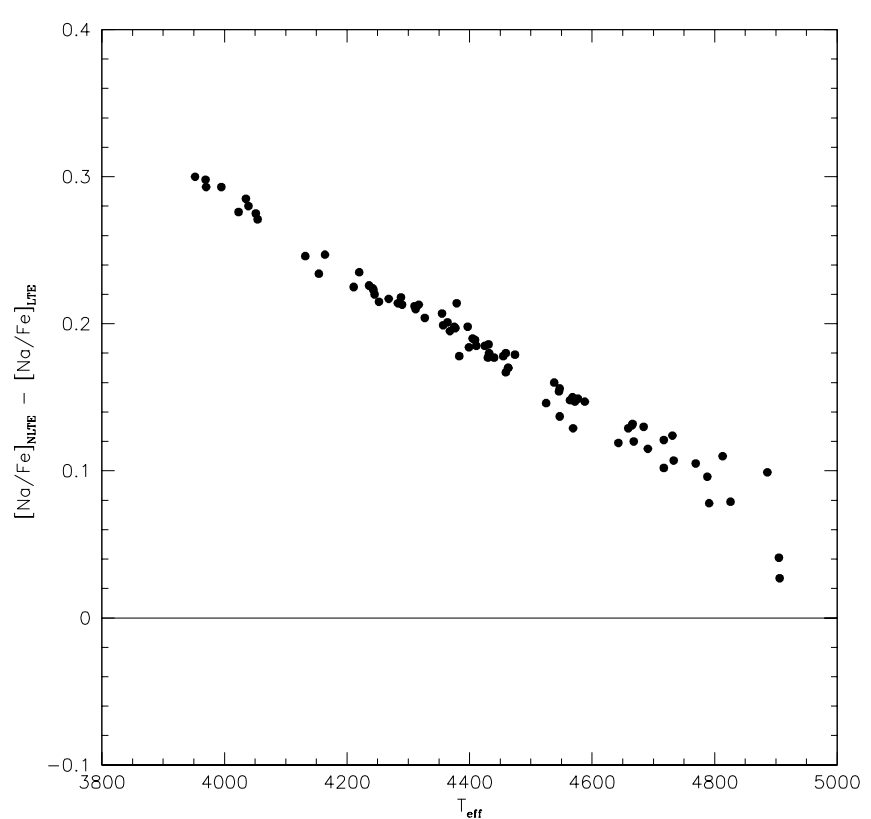

Fig. 5. Corrections to the $\mathrm{Na}$ abundances for departure from LTE as a function of effective temperature, derived for our program stars.

with tip giants having $0.2-0.3$ dex lower $[\mathrm{Na} / \mathrm{Fe}]$ abundances than less evolved RGB stars.

\section{Results and discussion}

Our final $[\mathrm{Na} / \mathrm{Fe}]$ abundances (including NLTE corrections) are plotted as a function of temperature in Fig. 6.

The first clear evidence that can be derived from this plot is the large star-to-star scatter, at every luminosity/temperature along the RGB. Also, the average $\mathrm{Na}$ abundance is generally quite high. Neither feature is surprising in itself, as both have been commonly found in several other clusters; now they are found for the first time also in this cluster, that had never been studied in detail from a spectroscopic point of view before.

When compared to abundances of field stars of similar metallicity (e.g. Gratton et al. 2000; Pilachowski et al. 1996a), these results confirm once more that globular cluster stars present a pattern of abundances suggestive of processes involving elements produced somewhere deep in the star, near the $\mathrm{H}$-burning shell, by proton-capture nucleosynthesis that operates at high temperatures, and likely producing $\mathrm{Na}$ through the NeNa cycle.

In fact, while field stars have generally low $\mathrm{Na}$ abundances (almost coincident with the lower envelope of the cluster abundances), with small scatter around the average value (comparable with observational errors), in cluster stars the standard evolutionary paradigm is broken. The spread in $\mathrm{Na}$ abundances in NGC 2808 reaches as much as 1 dex and does not decrease with temperature much below $0.5 \mathrm{dex}$, in $[\mathrm{Na} / \mathrm{Fe}]$.

Apart from the well known $\mathrm{Na}-\mathrm{O}$ anticorrelation (also present among RGB stars of NGC 2808 at a level that could be at least comparable with that existing in M 13, Carretta et al. 2003b), our results from $\mathrm{Na}$ abundances alone allow us to conclude that the standard first dredge-up and an additional mixing mechanism at luminosities above the RGB-bump are not

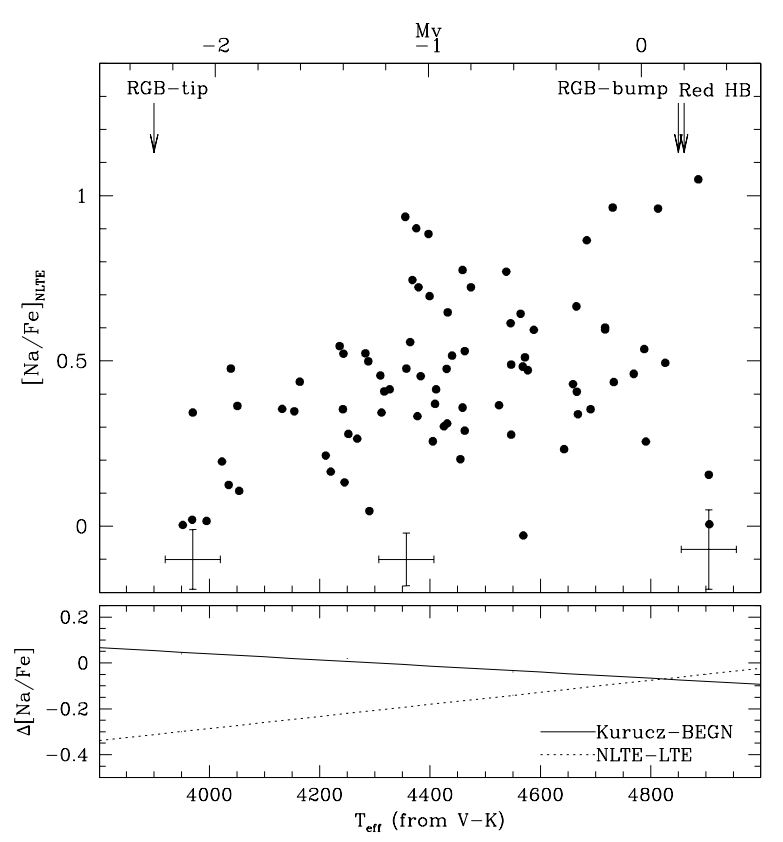

Fig. 6. Upper panel: abundance ratios $[\mathrm{Na} / \mathrm{Fe}]$ for stars along the $\mathrm{RGB}$ of NGC 2808, corrected for the effects of departure from LTE, as a function of the effective temperatures. At the top of the figure the approximate luminosity levels for the RGB-tip, the RGB-bump and the average luminosity of the red extreme of the $\mathrm{HB}$ are indicated. At the bottom of the figure we show the typical error bars over the whole range of temperatures spanned by our sample, due to random uncertainties in the adopted atmospheric parameters and measurement errors. Bottom panel: the solid line indicates the difference in $[\mathrm{Na} / \mathrm{Fe}]$ derived by using Bell et al. (1976) model atmospheres with respect to Kurucz (1995) models. The dashed line indicates the effect of NLTE vs. LTE approximations on the derived $[\mathrm{Na} / \mathrm{Fe}]$ abundances.

enough to explain the observed pattern, at odds with what happens in field stars.

$\mathrm{Na}$ abundances so large and scattered from star to star do require that a variable amount of Na-enriched matter polluted the stars in the past, or that the efficiency of some further (deep) mixing mechanism varies along the RGB modifying pre-existing stellar abundances. Thus, the question is now: is the observed scatter a cosmic scatter intrinsic to the studied sample, or is it an artifact of the analysis due to conjuring effects of uncertainties on derived abundances?

In general, we may consider three sources of errors on abundance determinations, some of which may affect the scatter, and some others only the zero point. First, the adopted temperature or oscillator strength scales may be wrong ${ }^{6}$. However, this would result only in a zero point shift, affecting rigidly the

\footnotetext{
${ }^{6}$ Note however that a systematic error of about $100 \mathrm{~K}$ in the temperature scale would give a difference of about 0.2 dex in abundances from neutral and singly ionized $\mathrm{Fe}$ lines, while the average difference found by Carretta et al. (2003b, from whom we adopted the average metallicity for NGC 2808) is only -0.01 dex, implying a rather small systematic error on the temperatures. Moreover, the atomic parameters for the strong $\mathrm{Na} \mathrm{D}$ lines are well known, as well as the laboratory oscillator strengths for many of the Fe lines used to derive the abundances from the UVES spectra.
} 
entire metallicity distribution but leaving almost unchanged the star-to-star spread.

Second, other effects exist, that may result in spurious systematic trends on the results if not taken into account. A clear example is provided by the corrections for departure from LTE considered in Sect. 4.1. In the bottom panel of Fig. 6 we have shown (dashed line) the changes in $[\mathrm{Na} / \mathrm{Fe}]$ abundances that would result by neglecting the non-LTE corrections. Since, as shown by Fig. 5 and discussed in Gratton et al. (1999), the amount of the correction increases at lower temperatures and gravities, had we neglected this effect a marked trend would appear in the results, with $\mathrm{Na}$ abundances decreasing as the stars approach the RGB-tip. Notice that this effect should be in principle larger at low metallicity. This should be borne in mind when comparing the pattern found in different clusters, such as the survey of $\mathrm{Na}$ abundances in giants of M 15 and M92 by Sneden et al. (2000) where Na D lines were also used, but no correction for departure from LTE was applied.

Another effect of some impact may be the choice of the grid of atmospheric models used in the analysis. In the present study we adopted the Kurucz (1995; K95) models with the overshooting option. We then repeated the analysis by computing the $\mathrm{Na}$ abundances using the Bell et al. (1976; hereinafter BEGN) model atmospheres, that are maybe a bit better at reproducing the atmospheres of cooler stars such as those at the RGB-tip. Figure 6 shows (bottom panel solid line) that at $T_{\text {eff }}$ about $3900 \mathrm{~K}$ the BEGN models provide $[\mathrm{Na} / \mathrm{Fe}]$ ratios about 0.04 dex larger than those derived with K95 models. At lower luminosity, around $T_{\text {eff }} \sim 4900 \mathrm{~K}$, the trend is inverted, with abundances from BEGN models being about 0.08 dex lower than those from K95. The net effect is again a (small) trend; if one used BEGN models and no corrections for non-LTE, the two opposite trends would nearly compensate each other. Anyway, also this effect would only tilt the distribution function of $\mathrm{Na}$ abundances along the RGB, leaving the rms scatter untouched.

Finally, random errors due to uncertainties in the adopted atmospheric parameters and to measurement errors may potentially add a random noise to the observed abundances. Are they enough to explain the observed scatter? In order to test this, we evaluated the sensitivity of abundances to errors in the adopted atmospheric parameters in the usual way, i.e. we repeated the analysis changing by a given amount one parameter at a time $\left(T_{\text {eff }}, \log g,[\mathrm{~A} / \mathrm{H}]\right.$ and $\left.v_{t}\right)$. We performed this test on three typical stars spaced all along the RGB, the results of this exercise are summarized in Table 2 .

To correctly use this table, one has to notice that the changes in the parameters are arbitrary, and have been adopted only for demonstrative purposes. For example, realistic errors in $T_{\text {eff }}$ are not likely to exceed $\sim 50 \mathrm{~K}$, whereas in Col. 2 of Table 2 we have used twice this value. Similarly, the gravity is unlikely to be wrong by much more than 0.1 dex and a conservative estimate of errors in metallicity is about 0.1 dex (Carretta et al. 2003b), hence the values listed in Cols. 3 and 4 should be scaled accordingly. Moreover, since our derivation of atmospheric parameters uses the position of stars in the CMD, errors in $T_{\text {eff }}$ and $\log g$ cannot be considered independent: e.g., an error in $T_{\text {eff }}$ of about $100 \mathrm{~K}$ would also correspond to an
Table 2. Sensitivities of $[\mathrm{Na} / \mathrm{Fe}]$ abundances to demonstrative errors in the atmospheric parameters.

\begin{tabular}{|c|c|c|c|c|}
\hline Element & $\begin{array}{r}\Delta T_{\text {eff }} \\
+100 \mathrm{~K} \\
\end{array}$ & $\begin{array}{r}\Delta \log g \\
+0.3 \mathrm{dex}\end{array}$ & $\begin{array}{l}\Delta[\mathrm{A} / \mathrm{H}] \\
0.2 \mathrm{dex}\end{array}$ & $\begin{array}{r}\Delta v_{t} \\
+0.2 \mathrm{~km} \mathrm{~s}^{-1} \\
\end{array}$ \\
\hline \multicolumn{5}{|c|}{ star 51871: $T_{\text {eff }}=3970 \mathrm{~K}, \log g=0.62$} \\
\hline$[\mathrm{Na} / \mathrm{Fe}]$ & +0.205 & -0.105 & +0.011 & -0.008 \\
\hline \multicolumn{5}{|c|}{ star 39060: $T_{\text {eff }}=4357 \mathrm{~K}, \log g=1.28$} \\
\hline$[\mathrm{Na} / \mathrm{Fe}]$ & +0.172 & -0.132 & +0.028 & -0.016 \\
\hline \multicolumn{5}{|c|}{ star 56136: $T_{\text {eff }}=4905 \mathrm{~K}, \log g=2.38$} \\
\hline$[\mathrm{Na} / \mathrm{Fe}]$ & +0.292 & -0.156 & +0.041 & -0.017 \\
\hline
\end{tabular}

uncertainty of about $0.25-0.30$ dex in $\log g$. So the contributions from these two terms must first be summed up algebrically, and the result be summed in quadrature along with all other independent contributions to give the final error due to uncertainties in all the adopted parameters. These typically range from 0.06 dex for the brightest stars in the sample up to 0.10 dex for the faintest stars observed at the RHB level.

To these, one has to add the uncertainties in the measurements. These random errors (including e.g. the continuum placement, the visual estimate of $\mathrm{Na}$ abundances from comparison with synthetic spectra) can be evaluated from the rms deviations of the average of $\mathrm{Na}$ abundances given by the two $\mathrm{Na} \mathrm{D}$ lines. They range typically from $0.05 \mathrm{dex}$ at the RGB-tip, to 0.06 dex around $4350 \mathrm{~K}$, and to $0.07 \mathrm{dex}$ for the faintest stars in the sample.

The resulting total random errors then vary from $0.08-$ 0.09 dex for brighter stars and increase to as much as $0.12 \mathrm{dex}$ at the RHB level. The random error bars are displayed in the upper panel of Fig. 6. Hence, the observed spread in Na abundances is more than 5 times the error for the bright part of the sample and as much as 8-10 times the error when considering the lower luminosity giants.

A first, firm result of these observations is then that large variations do exist in the abundances of elements produced in proton-capture reactions at all luminosities along the Red Giant Branch of NGC 2808, as shown by the large spread in $[\mathrm{Na} / \mathrm{Fe}]$ abundances.

Apart from specific computations, Fig. 7 allows to immediately appreciate how much $\mathrm{Na}$ can differ between pairs of stars having the same temperature, hence the same evolutionary physical status.

\section{Comparison with the $\mathrm{Na}$ distribution in other Globular Clusters}

The proton capture elements as Na in RGB stars have been studied in several GC's, mainly by the Lick and Texas groups. We may compare here our findings with what has been derived for the following GC's $([\mathrm{Fe} / \mathrm{H}]$ values are all on the Carretta \& Gratton 1997 metallicity scale): M 5 (Ivans et al. 2001; $[\mathrm{Fe} / \mathrm{H}]=-1.11$ ), M 13 (Pilachowski et al. 1996b; 


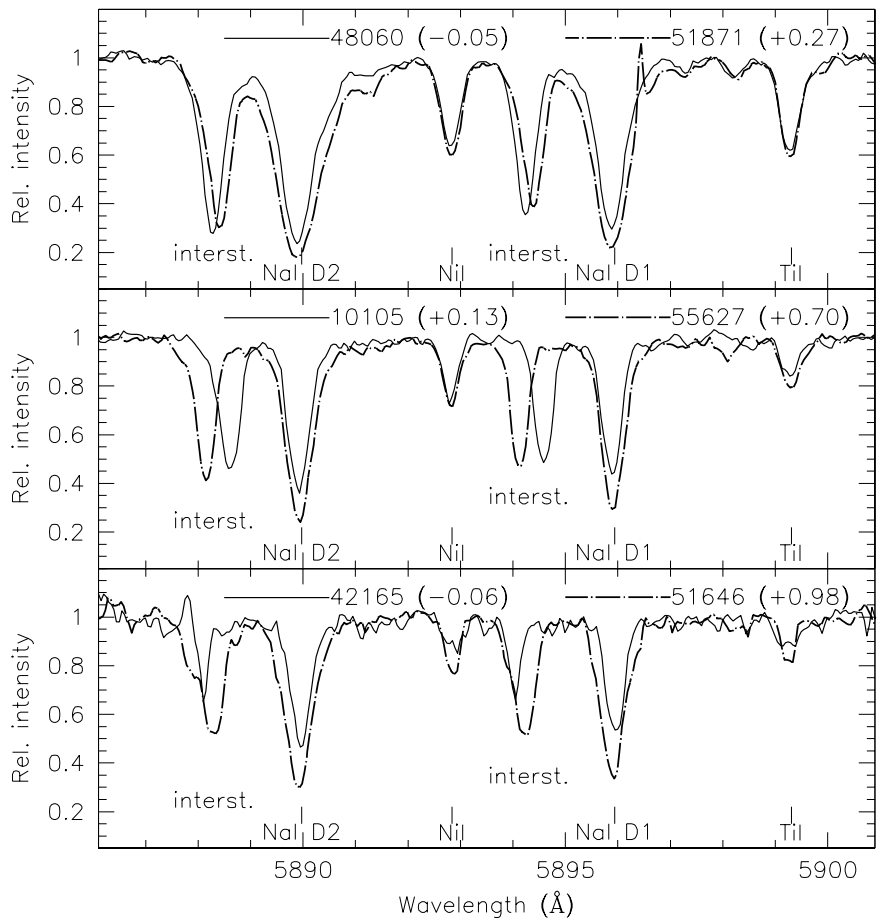

Fig. 7. Observed spectra of pairs of stars with different strenghts in $\mathrm{Na} \mathrm{D}$ lines, at the top $(3970 \mathrm{~K})$, in the middle $(4450 \mathrm{~K})$ and at the bottom (4906 K) of the temperature range spanned by our sample. Star identifications are given at the top of each panel, together with the $[\mathrm{Na} / \mathrm{Fe}]$ value. A few photospheric lines are indicated, while "interst." shows the position of the interstellar $\mathrm{Na} \mathrm{D}$ lines.

Kraft et al. 1997; $[\mathrm{Fe} / \mathrm{H}]=-1.39)$, M 15 and M 92 (Sneden et al. 2000; $[\mathrm{Fe} / \mathrm{H}]=-2.12$ and -2.16 , respectively). M 4, studied by Ivans et al. (1999), will not be considered here because severe differential reddening makes the separation of true RGB from AGB stars very difficult.

We have taken the $[\mathrm{Na} / \mathrm{Fe}]$ values from the above quoted papers, together with the photometric information that comes originally from: Sandquist et al. (1996, M5), Cudworth \& Monet (1979, M 13), Cudworth (1976, M 15), and Rees (1992, M 92). The distance moduli $(m-M)_{V}$ and reddenings were taken from Harris (1996). These $[\mathrm{Na} / \mathrm{Fe}]$ values come from moderately high $(R=11000)$ to very high $(R=60000)$ resolution spectra. The analyses differ from ours in some parts: in some cases $\mathrm{Na}$ abundances have been derived from the 5682-88 $\AA$ doublet (M 5 and M 13), in others from the $\mathrm{Na}$ D lines (M 15 and M 92); the Bell et al. (1976) grid of model atmosphere was usually used by the Texas-Lick group; finally, no correction for departure from LTE has been applied (this is slightly less important when the 5682-88 A lines are used).

\subsection{NGC 2808 and $M 13$}

The largest samples of RGB stars are for M 13 (112) and NGC 2808 (81), while less than about 30 RGB stars have been observed in the other GC's. The first comparison then will be done between these two GC's, since statistical significance and large sample of stars in different evolutionary phases are required to disentangle possible evolutionary effects, if any, overimposed to primordial variations. Moreover, Pilachowski et al. (1996b, hereafter PSKL) and Kraft et al. (1997) made a case for evolutionary effects being strongly at work in M 13, which is also the template cluster as far as extreme oxygen depletion in RGB stars is concerned.

The two clusters have been observed at similar resolutions ( $R \sim 11000$ and 15000 respectively for M 13 and NGC 2808), in both cases $\mathrm{Na}$ abundances come from spectral synthesis (5682-88 A lines, and Na D lines, respectively), the metallicities are quite similar ( 0.25 dex less for M 13), and only true RGB stars are considered in the comparison.

We note here that 13 more RGB tip stars were observed in NGC 2808, with UVES, and preliminary Na abundances were derived from abundance analysis based on the EW's of four lines, the subordinate lines 5862-88 $\AA$ and 6154-60 A. We do not include these stars in the present analysis to maintain a strict homogeneity in our database, however it's worth mentioning that they agree very well with the present results.

To evaluate the (possible) presence of evolutionary effects we have to compare samples of stars in different phases along the RGB. PSKL define as "RGB tip" stars those having $\log g \lesssim 1$, since they find an abrupt change in the Na abundance distribution at this point, with $[\mathrm{Na} / \mathrm{Fe}]$ becoming larger and less dispersed for higher luminosity stars. We find a similar separation, but in our case the Na abundances decrease in the RGB tip stars. While it is not clear if this point has some physical relevance, e.g. marking the onset of a particular regime when the star approaches the last stages of lifetime as a red giant, in the following we will adopt $\log g=1.02$ as separator (as done e.g., in M 5 by Ivans et al. 2001) between RGB-tip and lower-RGB stars (see also PSKL for a discussion on this point).

However, before attempting any comparison, we have to spend a few cautionary words on the various factors that may introduce spurious differences. When we compare results for bright and fainter objects, the larger random errors that are intrinsic to the analysis of fainter stars can produce an artificial increase in the abundance dispersion. Moreover, when we compare abundance analyses done by different groups we also have to take into account the different tools adopted: the use of the Kurucz or BEGN models for atmospheres, or the choice about correcting or not for NLTE, both introduce a tilt (see Fig. 6) that influences the median values of the Na distributions.

Figure 8 (lower panels) shows the distribution along the RGB's of the observed stars for NGC 2808 and M 13, in the dereddened $(B-V)_{0}, M_{V}$ plane, while in the upper panels are plotted the distributions of the $[\mathrm{Na} / \mathrm{Fe}]$ ratios for RGB tip and lower RGB stars; the vertical lines indicate the median value of each distribution. NGC 2808 and M 13 appear to behave quite differently in both bins/ranges. As already noted by PSKL, in M 13 the brightest stars are skewed towards high $\mathrm{Na}$ abundances (the median of the $[\mathrm{Na} / \mathrm{Fe}]$ values is $+0.41 \mathrm{dex}$ ) and show a small dispersion, while among the lower RGB stars there is a number of objects that are more Na-poor (median +0.16 dex) and with a larger dispersion. Notice that the - small - differential corrections (needed to translate abundances derived with BEGN models to our own scale) between the two bins would only increase the difference, shifting the medians apart. 

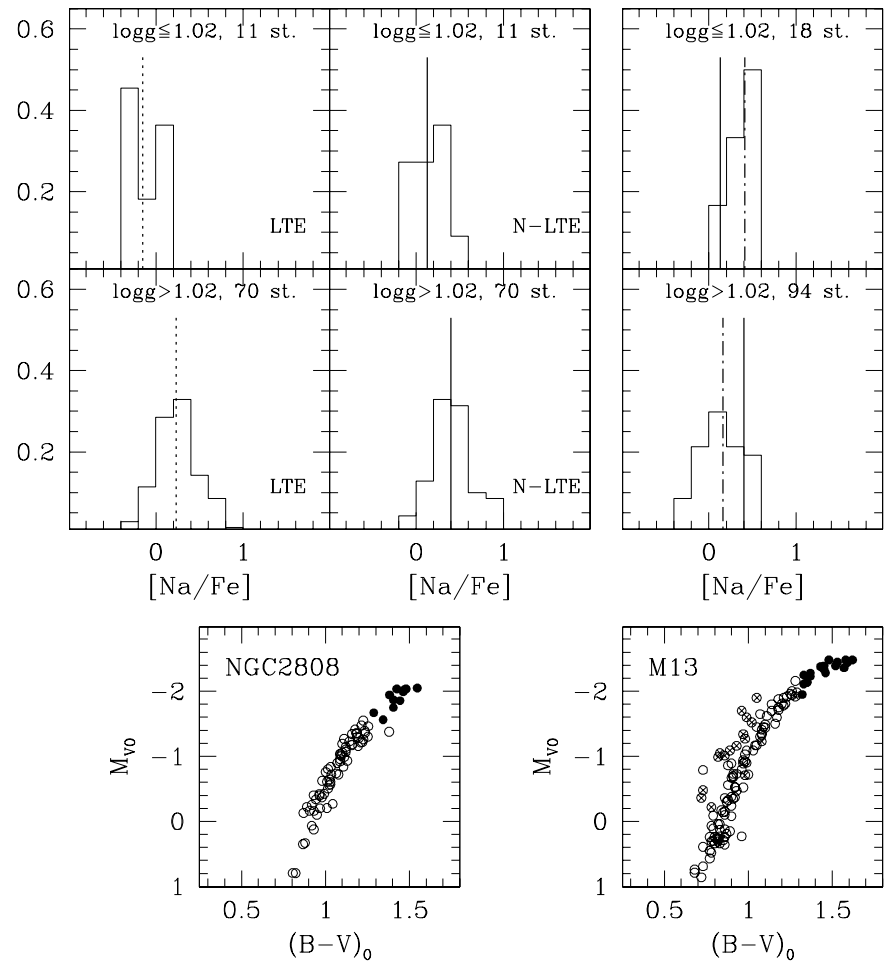

Fig. 8. Bottom panels: CMD's for the RGB stars studied in NGC 2808 and M 13; filled and open circles represent stars with $\log g \leq$ and $>1.02$, respectively; crosses indicate objects not considered in the histograms because they are possible AGB stars. Upper panels: histograms for the $[\mathrm{Na} / \mathrm{Fe}]$ values, for $\log g \leq$ and $>1.02$ respectively, normalized to the total number of stars used in the adopted range (labelled in the box). For NGC 2808 we plot both the LTE and NLTE cases. The vertical lines (dotted: NGC 2808 LTE; solid: NGC 2808 NLTE; dashed-dotted: M 13) indicate the median values for each considered range and case; the value for the NLTE distributions in NGC 2808 are shown also in the M 13 panels for comparison.

In NGC 2808 the opposite seems to be true: the RGB tip stars are Na-poorer, on average: a median value of $0.13 \mathrm{dex}$ is found, to be compared with 0.40 dex for lower luminosity RGB stars. When running simple Kolmogorov-Smirnov tests, these differences (between the two bins for the same GC, and between GC's) appear significant: the two ranges in $\log g$ (or luminosity and/or temperature) have very low probability of being extracted from the same parent population. Moreover, both ranges seem to be statistically different between M 13 and NGC 2808. While a larger spread at hotter temperatures is expected because of the worsening of spectra quality at lower luminosities, it is the median value that matters, as far as the interpretation of this data is concerned.

The interpretation by PSKL of this pattern of abundances in M 13 was that, in addition to the primordial variations among stars, an evolutionary effect is present, that increases the $\mathrm{Na}$ abundance somewhat differently for each star, since the mixing efficiency is different from stars to star. This evolutionary $\mathrm{Na}$ enhancement is dominant in the upper RGB, as witnessed also by the lowering of carbon isotopic ratios and carbon abundances (accompanied by increasing nitrogen abundances climbing up the RGB in several clusters, see the review by Kraft 1994 for references). Instead, in NGC 2808 this enhancement of the number of Na-rich stars is not seen, and the variation goes the opposite way, as in other clusters (see below).

The conclusions of this comparison are that:

(i) in NGC 2808 the evolutionary effect claimed in M 13 - if at all present - is only at the noise level. This would imply that the Na abundance distribution along the RGB is most probably of primordial origin, either because it was imprinted in the gas from which these stars originated, or because it was produced by an early pollution event due to a first generation of intermediate-mass AGB stars;

(ii) this finding seems to be quite at odds with what one may expect from the distribution of stars on the $\mathrm{HB}$, which is rather peculiar in NGC 2808 (an important example of "secondparameter" effect). Gratton (1982) already noted that about one fourth of the HB stars lie on the blue HB, which is quite unexpected given the metallicity of the cluster.

On the working hypothesis (recently explored by D'Antona et al. 2002) that Na-rich stars are also enriched in helium (as well as depleted in oxygen, as found in NGC 2808 by Carretta et al. 2003b), they would populate exactly the blue part of the HB. This, however, would require that about $1 / 4$ of the stars in the upper RGB stand out as Na-rich objects, which is maybe observed in M 13 but not very clearly in NGC 2808. The scenario proposed by D'Antona et al. (2002) also implies that more than one episode of star formation may have occurred within a GC, producing a bi(multi)-modal mass distribution on the HB. A similar type of distribution in the $\mathrm{Na}$ abundances should then be present along the RGB, which however is not observed with a sufficient degree of confidence.

In our opinion, the lack of a clear peak at high $\mathrm{Na}$ values in the distribution for NGC 2808 is an indication that the possible link between HB morphology (extension and mass distribution) and other parameters such as the extent of chemical anomalies may be not very tight.

\subsection{Comparison with other clusters: M5, M 15, M92}

As a result of the above comparison, can we deduce that NGC 2808 is a peculiar GC? Or is instead M 13 that behaves unusually? Already Ivans et al. (2001) in their study on M 5 noted that this cluster does not behave like M 13 (see their Fig. 11). To M 5 we may add M 15 and M 92: even with smaller numbers, the samples in these three GCs reach down to the same luminosity level $\left(M_{V} \sim 0\right)$ of the bulk of our program stars, so we feel entitled to repeat the comparison.

The results are displayed in Fig. 9. In all three cases the $[\mathrm{Na} / \mathrm{Fe}]$ distribution does not vary significantly (as deduced again by the Kolmogorov-Smirnov test) between the two bins (upper and lower RGB), and the median values are quite similar, in particular for the lower RGB. Again, based on this test, M 13 appears to stand out among globular clusters, since none of the examined clusters shows a clearcut shift toward Na-rich stars when climbing up the RGB. This is not surprising, as M 13 is peculiar in other ways, like its anomalously extendend 

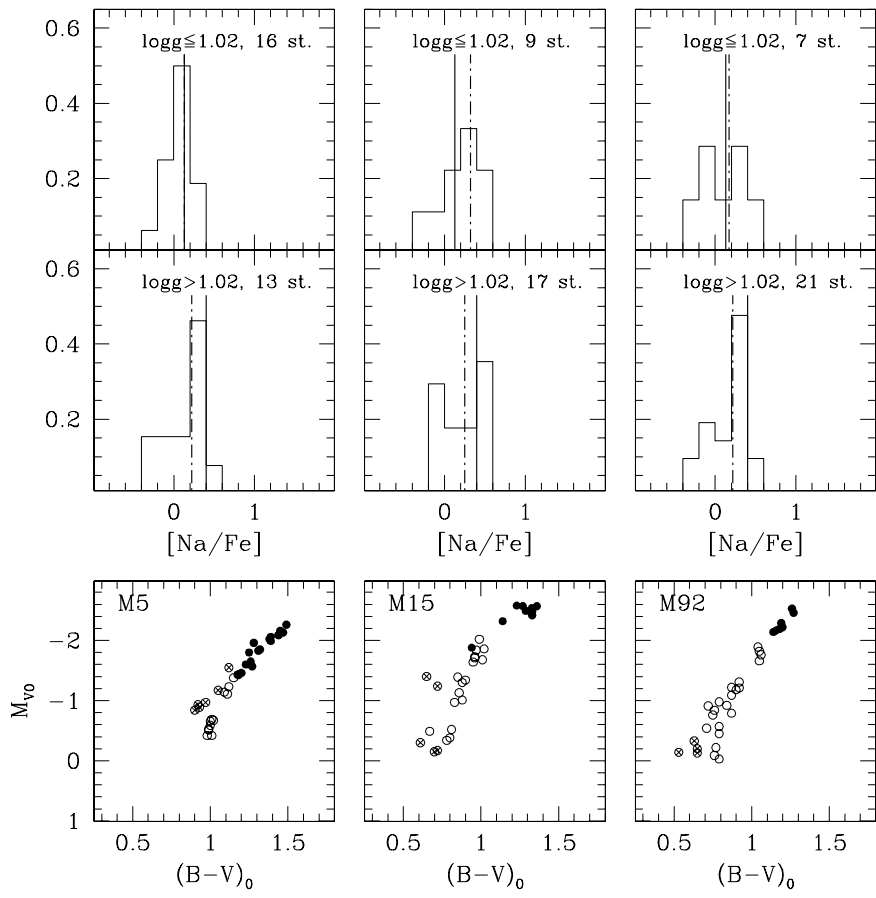

Fig. 9. The same as Fig. 8, but for M 5, M 15, and M 92.

$\mathrm{Na}-\mathrm{O}$ anticorrelation. It seems that in every other cluster, the scatter introduced by possible evolutionary effects is very small and does not affect sensibly the resulting abundances. This is more evident in the 3 other clusters shown in Fig. 9, where stars are observed preferentially on the upper part of the RGB. An increase of the scatter is hardly detectable.

\section{Summary}

We have analysed moderately high-resolution spectra of more than 80 red giant stars in the globular cluster NGC 2808, taken during the Science Verification program of the FLAMES multi-object spectrograph at the VLT. Atmospheric parameters were derived from the photometric information, radial velocities (which permitted to exclude a few field interlopers, leaving a sample of 81 RGB stars) were derived from the spectra. We examined in this paper only the sodium abundances, that were derived from the $\mathrm{Na}$ I $\mathrm{D}$ lines and spectral synthesis, and corrected for NLTE effects. The main results of our analysis are:

1 - There are large variations in $\mathrm{Na}$ abundances at all luminosity levels along the RGB in NGC 2808. This is an intrinsic spread, since it is much larger than possible uncertainties in the derived abundances (being from 0.5 to 1 dex, to compare with errors of about 0.08 dex increasing to 0.12 dex at the very faint end of our sample). While this is not surprising, being commonly observed in several globular clusters, this is the first time that detailed abundance analysis uncovers this pattern in NGC 2808. This spread is not explained by standard stellar evolutionary models, and its presence requires variable amount of yields from proton capture fusion on neon, that can only happen in the interior region near the H-burning shell, in the same star examined or in other objects that were then able to somehow pollute its surface.
2 - We have compared RGB-tip to lower-RGB stars to see whether there is any evolutionary effect acting like in M13, and enhancing $\mathrm{Na}$ for the brighter, more evolved giants. This effect does not appear to be important in NGC 2808, since brighter RGB stars have lower average $\mathrm{Na}$ abundances; if a similar mechanism is at work in this cluster, it only contributes adding some noise to a primordial dispersion. Our data are not sufficient to distinguish if the different composition was preexisting the star forming gas, or is due to pollution on the star surface.

3 - This Na abundance dispersion, and the (absence of) variation towards the RGB tip is similar to what was found for other GCs, e.g. M 5, M 15, and M92, but at odds with what is seen in M 13, the only other cluster for which a very large sample of RGB stars has been observed.

Homogeneous analysis of large samples of stars observed at high resolution and high $S / N$ is now possible for all Galactic globular clusters, with the new multiobject spectrographs mounted on $8-10 \mathrm{~m}$ class telescopes. This is the road we have to follow if we wish to make significative improvements on our knowledge of stellar formation and evolution in globular clusters.

Acknowledgements. This research has made use of data taken during FLAMES Science Verification. We wish to warmly thank Luca Pasquini for having co-ordinated the building of such a powerful instrument, and the ESO staff at Paranal for their excellent work during SV. We are specially indebted to Raffaele Gratton for many interesting and helpful suggestions and discussions that greatly improved the present work. We also thank the referee (Bob Rood) for his suggestions.

\section{References}

Alonso, A., Arribas, S., \& Martinez-Roger, C. 1999, A\&AS, 140, 261 Alonso, A., Arribas, S., \& Martinez-Roger, C. 2001, A\&A, 376, 1039 Bell, R. A., Eriksson, K., Gustafsson, B., \& Nordlund, A. 1976, A\&AS, 23, 37

Bragaglia, A., Carretta, E., Gratton, R. G., et al. 2001, AJ, 121, 327

Bruls, J. H. M., Rutten, R. J., \& Shchukina, N. G. 1992, A\&A, 265, 237

Cacciari, C., Bragaglia, A., Rossetti, E., et al. 2003, A\&A, in press

Cannon, R. D., Croke, B. F. W., Bell, R. A., Hesser, J. E., \& Stathakis, R. A. 1998, MNRAS, 298, 601

Cardelli, J. A., Clayton, G. C., \& Mathis, J. S. 1989, ApJ, 345, 245

Carretta, E., \& Gratton, R. G. 1997, A\&AS, 121, 95

Carretta, E., Gratton, R. G., Bragaglia, A., Bonifacio, P., \& Pasquini, L. 2003a, A\&A, submitted

Carretta, E., Bragaglia, A., Cacciari, C., Mulas, G., \& Rossetti, E. 2003b, in preparation

Cottrell, P. L., \& Da Costa, G. S. 1981, ApJ, 245, L79

Cudworth, K. M. 1976, AJ, 81, 519

Cudworth, K. M., \& Monet, D. G. 1979, AJ, 84, 774

D’Antona, F., Caloi, V., Montalban, J., Ventura, P., \& Gratton, R. G. 2002, A\&A, 395, 69

D'Antona, F., Gratton, R. G., \& Chieffi, A. 1983, MSAIt, 54, 173

Denisenkov, P. A., \& Denisenkova, S. N. 1990, Soviet Astron. Lett., 16,275

Gratton, R. G. 1982, A\&A, 115, 171

Gratton, R. G., Bonifacio, P., Bragaglia, A., et al. 2001, A\&A, 369, 87

Gratton, R. G., Carretta, E., Eriksson, K., \& Gustafsson, B. 1999, A\&A, 350, 955 
Gratton, R. G., Sneden, C., Carretta, E., \& Bragaglia, A. 2000, A\&A, 354,169

Harris, W. E. 1996, AJ, 112, 1487 (2003, update in http://physun.physics.mcmaster.ca/Globular.html)

Ivans, I., Kraft, R. P., Sneden, C., et al. 2001, AJ, 122, 1438

Ivans, I., Sneden, C., Kraft, R. P., et al. 1999, AJ, 118, 1273

Kraft, R. P. 1994, PASP, 106, 553

Kraft, R. P., Sneden, C., Smith, G. H., et al. 1997, AJ, 113, 279

Kurucz, R. L. 1995, CD-ROM 13, Smithsonian Astrophysical Observatory, Cambridge

Langer, G. E., Hoffman, R., \& Sneden, C. 1993, PASP, 105, 301

Lyons, M. A., Kemp, S. N., Bates, B., \& Shaw, C. R. 1996, MNRAS, 280,835

McWilliam, A., Preston, G. W., Sneden, C., \& Searle, L. 1995, AJ, Walker, A. R. 1999, AJ, 118, 432

Osborn, W. 1971, Observatory, 91, 223

Pasquini, L., Avila, G., Blecha, A., et al. 2002, The ESO Messenger, 110,1

Pilachowski, C. A., Sneden, C., \& Kraft, R. P. 1996a, AJ, 111, 1689

Pilachowki, C. A., Sneden, C., Kraft, R. P., \& Langer, G. E. 1996b, AJ, 112, 545

Rees, R. 1992, AJ, 103, 1573

Sandquist, E. L., Bolte, M., Stetson, P. B., \& Hesser, J. E. 1996, ApJ, 470,910

Smith, G. H. 1987, PASP, 99, 67

Sneden, C., Pilachowski, C. A., \& Kraft, R. P. 2000, 120, 1351

Ventura, P., D’Antona, F., Mazzitelli, I., \& Gratton, R. 2001, ApJ, 550, 65

109,2757 